\title{
A Contribution to Neutrosophic Groups
}

\author{
${ }^{1}$ Mohammad Abobala, Faculty of Science, Tishreen University, Lattakia, Syria \\ ${ }^{2}$ Ahmed Hatip, Department of Mathematics, Gaziantip University, Turkey \\ ${ }^{3}$ Riad K. Alhamido, Faculty of Science, Alfurat University, Deir Ezor, Syria \\ 1e-mail: mohammadabobala777@gmail.com \\ 2e-mail: kollnaar5@gmail.com \\ ${ }^{3}$ e-mail: riad-hamido1983@hotmail.com
}

\begin{abstract}
The objective of this paper is to define some new substructures (AH-substructures) in a neutrosophic group. Also, it deals with some elementary properties of AH-subgroups, $\mathrm{AH}$-normality, $\mathrm{AH}$-homomorphisms, $\mathrm{AH}$-quotients and AH-direct products.
\end{abstract}

Keywords: Neutrosophic group, AH- subgroup, AH- homomorphism, AH-quotient.

\section{Introduction}

The fuzzy set and intuitionistic fuzzy set theory were adopted effectively from their initiation to solve optimization problems at vague and uncertain situation in our daily life activities. The intuitionistic fuzzy set theory introduced by Atanassov [5] deals with the degree of belongingness and the degree of non-belongingness of an object to a set simultaneously. Thus it is the more generalization concept than fuzzy set theory which can provide only the degree of belongingness of an object to a set. Both of the theories can only handle incomplete information not indeterminate. To access both incomplete and indeterminate information, Smarandache [8,9] generalized the intuitionistic fuzzy set to neutrosophic set (NS) where each proposition is estimated by three independent parameters namely truth-membership value $(T)$, indeterminacy membership value $(I)$ and falsity-membership value $(F)$ with $T, I, F \in]-0,1+[$ and $-0 \leq \sup T+\sup I+\sup F \leq 3+$. Smarandache used to practice the standard or nonstandard subsets of $]-0,1+[$ in philosophical ground. So, to incorporate this concept in real life scenario, Wang et al. [10] brought the concept of single valued neutrosophic set which takes the value from real standard subset of [0,1] only.

Also, Smarandache and Vasantha Kandasamy introduced the notion of neutrosophic group in [1]. Neutrosophic rings were introduced in [4]. The neutrosophic group in general does not have a group structure. 
In this paper, we shall continue the study of neutrosophic groups by introducing the notion of an AH-subgroup and develop some basic theory, we will use the idea of AH-concepts introduced in $[1,2]$.

Neutrosophic AH-subgroup will be defined as a union of two subgroups $K \cup T ; \mathrm{K}$ is a subgroup of $\mathrm{G}$ and $\mathrm{T}$ is a subgroup of the pure neutrosophic group GI.

\section{Preliminaries}

In this following section, we recall some important and useful definitions about neutrosophic groups.

Definition 2.1: [3]

Let $\left(\mathrm{G},{ }^{*}\right)$ be a group . Then the neutrosophic group is generated by $\mathrm{G}$ and I under * denoted by

$\mathrm{N}(\mathrm{G})=\{<G \cup I>, *\}$

$I$ is called the indeterminate (neutrosophic element) with the property $I^{2}=I$.

Definition 2.2: [3]

Let $N(G)$ be a neutrosophic group and $H$ be a neutrosophic subgroup, i.e., (H contains a proper subgroup of $G$ ). Then $\mathrm{H}$ is a neutrosophic normal subgroup of $\mathrm{N}(\mathrm{G})$ if $x H=H x$ for all $x \in N(G)$.

\section{Definition 2.3: [3]}

Let $N(G)$ be a neutrosophic group. Then the center of $N(G)$ is denoted by $C(N(G))$, and defined

$C(N(G))=\{x \in N(G) ; x y=y x \forall y \in N(G)\}$

Definition 2.4: [3]

Let $\mathrm{N}(\mathrm{G}), \mathrm{N}(\mathrm{H})$ be two neutrosophic groups, then $N(G) \times N(H)=\{(g, h) ; g \in N(G), h \in N(H)\}$.

Definition 2.5: [6]

Let $\mathrm{N}(\mathrm{G}), \mathrm{N}(\mathrm{H})$ be two neutrosophic groups and $\varphi: N(G) \rightarrow N(H)$ is called a neutrosophic homomorphism if it is a homomrphism between $\mathrm{G}, \mathrm{H}$ and $\varphi(I)=I^{\prime}$.

Where $I^{\prime}$ is the neutrosophic element of $\mathrm{N}(\mathrm{H})$.

If $\varphi$ is a correspondence one-to-one it is called a neutrosophic isomorphism.

\section{Example 2.6:}

Let $Z_{7}=\{0,1,2, \ldots, 6\}$ be the group of integers under addition modulo $7 . N(G)=\left\{\left\langle Z_{7} \cup \mathrm{I}\right\rangle\right.$, ' + ' modulo 7$\}$ is a neutrosophic group which is in fact a group. For $N(G)=\left\{a+b I ; a, b \in Z_{7}\right\}$ is a group under ' + ' modulo 7. Thus this neutrosophic group is also a group.

\section{Theorem 2.7: [3]}

Let $\left(\mathrm{G},{ }^{*}\right)$ be a group, $\mathrm{N}(\mathrm{G})=\{\langle\mathrm{G} \cup \mathrm{I}\rangle, *\}$ be the neutrosophic group. Then 
$\mathrm{N}(\mathrm{G})$ in general is not a group.

$\mathrm{N}(\mathrm{G})$ always contains a group.

\section{Example 2.8:}

Let $\mathrm{N}(\mathrm{G})$ be a neutrosophic group generated by $\left(Z_{7}^{*},.\right)$, given by $\mathrm{N}(\mathrm{G})=\{1,2,3,4,5,6, \mathrm{I}, 2 \mathrm{I}, 3 \mathrm{I}, 4 \mathrm{I}, 5 \mathrm{I}, 6 \mathrm{I}\}$ be a neutrosophic group under multiplication modulo 7 .

$H=\{1,6, I, 6 I\}$ is a neutrosophic subgroup of $N(G)$. For $x \in N(G), x H=H x$, so $H$ is normal in $N(G)$.

\section{Definition 2.9: [11]}

(a) Let $\mathrm{G}$ be any group. It is called meta abelian if it has a abelian derivative subgroup $G^{\prime}$.

(b) Let $\mathrm{G}$ be a group. It is called nilpotent if it has a central series.

For the concept of central series, see [11].

\section{Remark 2.10: [11]}

$S_{3}$ is a solvable group, but it is not nilpotent.

$D_{4}$ is a meta abelian and nilpotent group.

The intersection, and the direct product of two meta abelian groups is meta abelian.

The intersection, and the direct product of two nilpotent groups is nilpotent.

AH-subgroups were firstly defined in n-refined neutrosophic groups. We recall some definitions.

\section{Definition 2.11: [1]}

Let $(G, *)$ be a group, we define the corresponding n-refined neutrosophic group $N_{n}(G)$ as follows:

$N_{n}(G)=\left(<G \cup\left\{I_{1}, \ldots, I_{n}\right\}>, *\right)=\left\{\left(a_{0}, a_{1} I_{1}, \ldots, a_{n} I_{n}\right) ; a_{i} \in G\right\}$.

It is easy to see that $N_{n}(G)$ is closed under $*$, and it is a semigroup but not a group since $I_{i}$ has no inverse with respect to $*$ in general.

\section{Remark 2.12: [1]}

If $(\mathrm{G},+)$ is an additive abelian group, then addition on $N_{n}(G)$ can be described as follows:

Consider $x=\left(a_{0}, a_{1} I_{1}, \ldots, a_{n} I_{n}\right), y=\left(b_{0}, b_{1} I_{1}, \ldots, b_{n} I_{n}\right)$, we have

$x+y=\left(a_{0}+b_{0},\left[a_{1}+b_{1}\right] I_{1}, \ldots,\left[a_{n}+b_{n}\right] I_{n}\right)$. In this case $\left(N_{n}(G),+\right)$ is a classical abelian group.

The identity element is $(0,0, \ldots, 0)$.

It is easy to see that $N_{n}(G) \cong G \times G \times . . \times G(n+1$ times $)$ in the case of abelian additive group $\mathrm{G}$.

\section{Remark 2.13: [1]}

If $\mathrm{G}$ is a multiplicative group, then group product on $N_{n}(G)$ can be described as follows: 
Consider $x=\left(a_{0}, a_{1} I_{1}, \ldots, a_{n} I_{n}\right), y=\left(b_{0}, b_{1} I_{1}, \ldots, b_{n} I_{n}\right)$, we have

$x y=\left(t_{0}, t_{1}, \ldots, t_{n}\right) ; t_{s}=\prod_{i, j=0}^{n}\left(a_{i} b_{j}\right) I_{i} I_{j} ; I_{0}=e_{G}$ and $I_{i} I_{j}=I_{s}$.

The identity element is $\left(e_{G}, e_{G} I_{1}, \ldots, e_{G} I_{n}\right)$.

In this case $N_{n}(G)$ is not isomorphic to the direct product of $\mathrm{n}+1$ copies of $\mathrm{G}$, since it is not a classical group in this case.

The binary operation between the sub-indeterminacies is $I_{i} \cdot I_{j}=I_{\min (i, j)}$.

\section{Main discussion}

Definition 3.1 :

Let $(G, *)$ be any group, the pure neutrosophic group $G I$ is the set $\{x * I$ :for every $x$ from $G\}$.

\section{Example 3.2 :}

Let $G=\left(Z_{5},+\right)$, then the corresponding pure neutrosophic group is

$\{0+I, 1+I, 2+I, 3+I, 4+I\}$, since $\left(^{*}\right)$ is considered as $(+)$ between the elements of $\mathrm{G}$.

\section{Theorem 3.3 :}

The set GI has a group structure under the binary operation $(\mathrm{xI})(\mathrm{yI})=(\mathrm{xy}) \mathrm{I}$ with identity $\mathrm{I}$ and $f: G \rightarrow G I$;

$f(x)=x I$ is an isomorphism.

Proof :

It is easy to see that GI is a group under the previous operation with identity I.

We have $f(x y)=x y I=(x I)(y I)=f(x) f(y)$, so $f$ is a homomorphism, and $f$ is a one to one correspondence, thus $f$ is an isomorphism clearly.

\section{Remark 3.4 :}

For every subgroup $\mathrm{H}$ of G, we can find an isomorphic subgroup with form HI of GI.

\section{Definition 3.5 :}

Let $\mathrm{N}(\mathrm{G})$ be a neutrosophic group and $\mathrm{K}$ be a subset of $\mathrm{N}(\mathrm{G})$, we say that $\mathrm{K}$ is an AH-subgroup if $\mathrm{K}=H \cup T$ such that $\mathrm{H}$ is a subgroup of $\mathrm{G}$ and $\mathrm{T}$ is a subgroup of $\mathrm{GI}$.

\section{Example 3.6 :}

Let $G=Z_{4}$, then $\mathrm{K}=\{0,2, I\}$ is an AH-subgroup of $\mathrm{N}(\mathrm{G})$ because $\{0,2\}$ is a subgroup of $\mathrm{G}$ and $\{\mathrm{I}\}$ is a subgroup of the pure neutrosophic group GI under the operation defined in Theorem 3.3.

\section{Definition 3.7 :}

Let $\mathrm{N}(\mathrm{G})$ be a neutrosophic group, $\mathrm{K}$ be an AH-subgroup, we say that $\mathrm{K}$ is AHS-subgroup if $\mathrm{T} \cong \mathrm{H}$. 


\section{Definition 3.8 :}

Let $\mathrm{N}(\mathrm{G})$ be a neutrosophic group, $\mathrm{K}$ be an $\mathrm{AH}$-subgroup we say that $\mathrm{K}$ is an $\mathrm{AH}$-normal subgroup if $\mathrm{H}$ is normal in $\mathrm{G}$ and $\mathrm{T}$ is normal in GI.

\section{Definition 3.9 :}

(a) Let $\mathrm{N}(\mathrm{G})$ be a neutrosophic group and $\mathrm{K}$ be an AHS-subgroup, we say that $\mathrm{K}$ is an AHS-normal subgroup if it is AH-normal.

(b) Let N(G) be a neutrosophic group, $K=K_{1} \cup K_{2}, S=S_{1} \cup S_{2}$ be two AH-subgroups. The intersection is defined as $K \cap S=\left(K_{1} \cap S_{1}\right) \cup\left(K_{2} \cap S_{2}\right)$.

Theorem 3.10 :

Let $\mathrm{N}(\mathrm{G})$ be a neutrosophic group, then

(a) If K, S are two AH-subgroups, $K \cap S$ is $\mathrm{AH}$-subgroup.

(b) If K, S are two AHS-subgroups, $K \cap S$ is AHS-subgroup.

(c) If $\mathrm{K}, \mathrm{S}$ are two AH-normal subgroups, $K \cap S$ is AH-normal subgroup.

(d) If K, $\mathrm{S}$ are two AHS-normal subgroups, $K \cap S$ is AHS-normal subgroup.

\section{Proof :}

(a) Suppose that $K=K_{1} \cup K_{2}, S=S_{1} \cup S_{2}$, then $K \cap S=\left(K_{1} \cap S_{1}\right) \cup\left(K_{2} \cap S_{2}\right)$ is an AH-subgroup, because $K_{1} \cap S_{1}$ is a subgroup of $\mathrm{G}$ and $K_{2} \cap S_{2}$ is a subgroup of GI.

(b) The proof holds directly from (a).

(c) If $K_{1}, S_{1}$ are normal subgroups of $\mathrm{G}$ and $K_{2}, S_{2}$ are normal in GI, then $K_{2} \cap S_{2}$ is normal in GI and $K_{1} \cap S_{1}$ is normal in G, thus $K \cap S=\left(K_{1} \cap S_{1}\right) \cup\left(K_{2} \cap S_{2}\right)$ is AH-normal subgroup.

(d) It holds from (c).

\section{Definition 3.11 :}

Let $\mathrm{N}(\mathrm{G})$ be a neutrosophic subgroup with two AH-subgroups $\mathrm{K}=K_{1} \cup K_{2}, \mathrm{~S}=S_{1} \cup S_{2}$.

We define $\mathrm{KS}=K_{1} S_{1} \cup K_{2} S_{2}$

\section{Theorem 3.12 :}

Let $\mathrm{N}(\mathrm{G})$ be a neutrosophic subgroup with two AH-normal subgroups $\mathrm{K}=K_{1} \cup K_{2}, \mathrm{~S}=S_{1} \cup S_{2}$, then $\mathrm{KS}$ is an AHnormal subgroup.

\section{Proof :}

Since $K_{1} S_{1}$ is normal subgroup of $\mathrm{G}$ and $K_{2} S_{2}$ is normal in GI. The proof is complete.

\section{Definition 3.13:}


Let $\mathrm{N}(\mathrm{G})$ be a neutrosophic subgroup with an AH-normal subgroup $\mathrm{K}=K_{1} \cup K_{2}$. We define the AH-Quotient $\mathrm{N}(\mathrm{G}) / \mathrm{K}=G / K_{1} \cup G I / K_{2}$.

If $\mathrm{K}$ is an AHS-normal subgroup, then $\mathrm{N}(\mathrm{G}) / \mathrm{K}$ is called AHS-Quotient.

The AHS-Quotient $\mathrm{N}(\mathrm{G}) / \mathrm{K}$ must be understood as $G / K_{1} \cup\left(G / K_{1}\right) I$ because $K_{1} \cong K_{2}$.

\section{Remark 3.14 :}

If $\mathrm{K}=K_{1} \cup K_{2}$ and $\mathrm{S}=S_{1} \cup S_{2}$ are two AH-subgroups, we say that $K \cong S$ if and only if $K_{1} \cong S_{1}$ and $K_{2} \cong S_{2}$.

\section{Theorem 3.15 :}

Let $\mathrm{N}(\mathrm{G})$ be a neutrosophic group with an AH-normal subgroup K. Then

(a) If $\mathrm{N}(\mathrm{G})$ is abelian, then $\mathrm{N}(\mathrm{G}) / \mathrm{K}$ is abelian.

(b) If $\mathrm{K}$ is an AHS-normal subgroup, and $x K=y K$ for $x, y \in G$, then $x y^{-1} \in K_{1}$. Also, if $y=z I \in G I$, then $x z^{-1} \in$ $K_{1}$.

(c) If $\mathrm{G}$ is finite and $\mathrm{K}$ is AHS-subgroup, $\mathrm{o}(\mathrm{K})$ will divide $\mathrm{o}(\mathrm{N}(\mathrm{G})$ ).

\section{Proof :}

(a) It can be proved as the classical case.

(b) Suppose that $\mathrm{y} \in G$, then by the proposition we have $x K_{1} \cup x I K_{2}=y K_{1} \cup y I K_{2}$, so $x K_{1}=y K_{1}$, thus $x y^{-1} \in K_{1}$.

Now if $y=z I \in G I$, then $x K_{1} \cup x I K_{2}=z K_{1} \cup z I K_{2}$, hence $x K_{1}=z K_{1}$, thus $x z^{-1} \in K_{1}$.

(c) It holds directly from Lagrange's theorem.

\section{Example 3.16 :}

Let $G=Z_{6}$ be the group of integers modulo 6 with respect to addition, we have $\{0,2,4, \mathrm{I}, 3+\mathrm{I}\}$ is an AH-normal subgroup, because $\{0,2,4\}$ is normal in $\mathrm{G}$ and $\{\mathrm{I}, 3+\mathrm{I}\}$ is normal in GI. The corresponding AH-quotient is

$\{1+\{0,2,4\},\{0,2,4\},\{I, 3+I\},(1+I)+\{I, 3+I\},(2+I)+\{I, 3+I\}\}$.

$\{0,3, \mathrm{I}, 3+\mathrm{I}\}$ is an AHS-normal subgroup of $\mathrm{N}(\mathrm{G})$ and the related AHS-quotient is

$\{\{0,3\}, 1+\{0,3\}, 2+\{0,3\},\{\mathrm{I}, 3+\mathrm{I}\},(1+\mathrm{I})+\{\mathrm{I}, 3+\mathrm{I}\},(2+\mathrm{I})+\{\mathrm{I}, 3+\mathrm{I}\}\}$.

\section{Theorem 3.17 :}

Let $\mathrm{N}(\mathrm{G})$ be a neutrosophic group, $\mathrm{K}$ be an AH-normal subgroup, $\mathrm{S}$ be an AH-subgroup of $\mathrm{N}(\mathrm{G}) / \mathrm{K}$, then there is an AH-subgroup $\mathrm{T}$ of $\mathrm{N}(\mathrm{G})$ such that $\mathrm{S}$ is contained in $\mathrm{T}$ as an $\mathrm{AH}$-normal subgroup.

\section{Proof :}

Suppose that $\mathrm{S}$ is an AH-subgroup of $\mathrm{N}(\mathrm{G}) / \mathrm{K}$, then $S=S_{1} \cup S_{2}$ such $S_{1}$ is a subgroup of $G / K_{1}$ and $S_{2}$ is a subgroup of $G I / K_{2}$, so that $S_{1}=T_{1} / K_{1}, S_{2}=T_{2} / K_{2}$ where $T_{1}, T_{2}$ are two subgroups of G, GI respectively, and $K_{1} \leq T_{1}, K_{2} \leq T_{2}$, we put $T=T_{1} \cup T_{2}$, thus we get the proof. 


\section{Definition 3.18 :}

Let $\mathrm{N}(\mathrm{G}), \mathrm{N}(\mathrm{H})$ be two neutrosophic groups with neutrosophic elements $I, I^{\prime}$ respectively, we define the AH-direct product $N(G) \times N(H)$ as a union

$(G \times H) \cup\left(G \times H I^{\prime}\right) \cup(G I \times H) \cup\left(G I \times H I^{\prime}\right)$.

For more comprehension of AH-structures we shall introduce the following definition.

\section{Definition 3.19 :}

Let $\mathrm{N}(\mathrm{G}), \mathrm{N}(\mathrm{H})$ be two neutrosophic groups and $f_{G}: G \rightarrow H$ be a homomorphism, we define the corresponding AHShomomorphism as follows:

$f: N(G) \rightarrow N(H) ; f(x+y I)=f_{G}(x)+f_{G}(y) I$.

We define $A H-\operatorname{Ker}(f)=\operatorname{Ker}\left(f_{G}\right) \cup \operatorname{Ker}\left(f_{G I}\right)$. It is easy to see that $\operatorname{Ker} f_{G} \cong \operatorname{Ker} f_{G I}$, so that $A H-\operatorname{Ker}(f)$ is an AHS-subgroup of $\mathrm{N}(\mathrm{G})$.

We can understand the AH-Kernel as a union, $A H-\operatorname{ker}(f)=\operatorname{Kerf} f_{G} \cup \operatorname{Kerf}_{G} I$.

\section{Theorem 3.20 :}

Let $\mathrm{f}$ be an AHS-homomorphism between $\mathrm{N}(\mathrm{G})$ and $\mathrm{N}(\mathrm{H})$. We have

(a) $A H-\operatorname{Ker}(f)$ is an AHS-normal subgroup of $\mathrm{N}(\mathrm{G})$.

(b) $\mathrm{N}(\mathrm{G}) / A H-\operatorname{Ker}(f) \cong f_{G}(G) \cup f_{G}(G) I$. (The isomorphism here is taken by the concept of AHS-isomorphism).

The quotient in (b) is taken as AH-quotient.

\section{Proof :}

(a) Since $\operatorname{Ker}\left(f_{G}\right)$ and $\operatorname{Ker}\left(f_{G I}\right)$ are normal in G, GI respectively, $A H-\operatorname{Ker}(f)$ is AHS-normal.

(b) We have $G / \operatorname{Ker} f_{G} \cong f_{G}(G)$ and $G I / \operatorname{Ker} f_{G I} \cong f_{G I}(G I)$,

$$
f(N(G))=f(G) \cup f(G I) \cong G / K \operatorname{Ker}\left(f_{G}\right) \cup G I / \operatorname{Ker}\left(f_{G I}\right)=\mathrm{N}(\mathrm{G}) / A H-\operatorname{Ker}(f) .
$$

\section{Theorem 3.21 :}

Let $\mathrm{N}(\mathrm{G})$ be a neutrosophic group and $K=K_{1} \cup K_{2}, H=H_{1} \cup H_{2}$ be two neutrosophic AHS-normal subgroups with $H \leq K$. Then

$(N(G) / H) /(K / H) \cong N(G) / K .($ Quotients and isomorphisms are taken as AH-concept).

\section{Proof :}

We have $(N(G) / H) /(K / H)=\left(G \cup G I / H_{1} \cup H_{2}\right) /\left(K_{1} \cup K_{2} / H_{1} \cup H_{2}\right)=G / H_{1} \cup\left(G I / H_{2}\right) /\left(K_{1} / H_{1}\right) \cup\left(K_{2} / H_{2}\right)$. So $(N(G) / H) /(K / H) \cong G / H_{1} /\left(K_{1} / H_{1}\right) \cup\left(G I / H_{2}\right) /\left(K_{2} / H_{2}\right) \cong G / K_{1} \cup G I / K_{2}=N(G) / K$.

\section{Theorem 3.22:}


Let $\mathrm{N}(\mathrm{G}), \mathrm{N}(\mathrm{H})$ be two neutrosophic groups with two AHS-normal subgroups $K=K_{1} \cup K_{2}, S=S_{1} \cup S_{2}$ respectively, Then

$N(G) \times N(H) / K \times S \cong N(G) / K \times N(H) / S$. (Direct products and isomorphisms are taken as AH-concepts).

\section{Proof :}

We have $N(G) \times N(H) / K \times S=$ $(G \times H) \cup\left(G \times H I^{\prime}\right) \cup(G I \times H) \cup\left(G I \times H I^{\prime}\right) /\left(K_{1} \times S_{1}\right) \cup\left(K_{1} \times S_{2}\right) \cup\left(K_{2} \times S_{1}\right) \cup\left(K_{2} \times S_{2}\right)=$ $\left(G \times H / K_{1} \times S_{1}\right) \cup\left(G \times H I^{\prime} / K_{1} \times S_{2}\right) \cup\left(G I \times H / K_{2} \times S_{1}\right) \cup\left(G I \times H I^{\prime} / K_{2} \times S_{2}\right) \cong N(G) / K \times N(H) / S$.

We will construct some examples to clarify the concepts defined previously.

\section{Example 3.23:}

Let $G=(Z,+), H=\left(Z_{12},+\right)$ be two groups, $N(G), N(H)$ be their corresponding neutrosophic groups,

$f_{G}: Z \rightarrow Z_{12} ; f(x)=x \bmod 12$ be a homomorphism, $\operatorname{Ker}\left(f_{G}\right)=12 Z, G I=G+I=\{x+I ; x \in Z\}$, since the considered operation in $\mathrm{G}$ is addition, $H I=H+I=\left\{x+I ; x \in Z_{12}\right\}$, since the considered operation in $\mathrm{H}$ is addition modulo 12 .

(a) $S_{1}=3 Z, S_{2}=6 Z+I=\{6 x+I ; x \in Z\}$ are two subgroups of G, G+I respectively. $K=S_{1} \cup S_{2}$ is an AHsubgroup of $\mathrm{N}(\mathrm{G})$.

(b) $f: N(G) \rightarrow N(H) ; f(x+y I)=f_{G}(x)+f_{G}(y) I=(x \bmod 12)+(y \bmod 12) I$ is an AH-homomorphism.

(c) $A H-\operatorname{Ker}(f)=\operatorname{Ker}\left(f_{G}\right) \cup \operatorname{Ker}\left(f_{G}\right) I=12 Z \cup(12 Z+I)$ which is an AHS-subgroup.

(d) The AH-quotient

$N(G) / A H-K \operatorname{Ker}(f)=G / 12 Z \cup[G / 12 Z+I] \cong Z_{12} \cup\left[Z_{12}+I\right]=H \cup[H+I]=f_{G}(G) \cup f_{G}(G) I$.

(d) $f(K)=f_{G}\left(S_{1}\right) \cup f_{G}\left(S_{2}\right)=\{0,3,6,9\} \cup(\{0,6\}+I)=\{0,3,6,9, I, 6+I\}$ which is an AH-subgroup of N(H).

(e) $K, f(K)$ are AH-normal, since commutavity implies normality in abelian groups.

(f) The AH-direct product of N(H) with itself is equal to

$N(H) \times N(H)=(H \times H) \cup(H \times H I) \cup(H I \times H) \cup(H I \times H I)$, according to Definition 3.18.

\section{Example 3.24:}

Let $G=Z_{12}$ be the group of integers modulo $6, H=\{0,2,4,6,8,10\}, K=\{0,3,6,9\}, S=\{0,6\}$ are normal subgroups of $\mathrm{G}$.

$M=H \cup K I=\{0,2,4,6,8,10\} \cup(\{0,3,6,9\}+I)=\{0,2,4,6,8,10, I, 3+I, 6+I, 9+I\}$ is an AH-normal subgroup, $N=K \cup S I=\{0,3,6,9\} \cup(\{0,6\}+I)=\{0,3,6,9, I, 6+I\}$ is another AH-normal subgroup, we clarify Theorem 3.12 as follows:

$M N=H K \cup K S I=\{h+k ; h \in H, k \in K\} \cup(\{k+s ; k \in K, s \in S\}+I)=$

$\{0,1,2,3,4,5,6,7,8,9,10,11\} \cup\{I, 3+I, 6+I, 9+I\}=\{0,1,2,3,4,5,6,7,8,9,10,11, I, 3+I, 6+I, 9+I\}$, which is an AH-normal subgroup. 
For a future research, we will show some definitions and new concepts related to AH-structures, such as AHsolvability, AH-nilpotency, and AH-cyclicity.

\section{Definition 3.25:}

Let $N(G)$ be a neutrosophic group, $N(H)=T \cup S ; T \leq G, S \leq G I$ be an AH-subgroup, we say

(a) $N(H)$ is an AH-solvable subgroup if $T, S$ are solvable.

(b) $N(H)$ is an AH-nilpotent subgroup if $T, S$ are nilpotent.

(c) $N(H)$ is an AH-abelian subgroup if $T, S$ are abelian.

(d) $N(H)$ is an AH-cyclic subgroup if $T, S$ are cyclic.

\section{Example 3.26:}

Let $G=S_{3}, T=<\left(\begin{array}{ll}1 & 2\end{array}\right)>, S=<\left(\begin{array}{lll}1 & 2 & 3\end{array}\right)>$ be two subgroups of $\mathrm{G}$, we have

(a) $S I=\left\{\left(\begin{array}{lll}1 & 2 & 3 \\ 2 & 3 & 1\end{array}\right) I,\left(\begin{array}{lll}1 & 2 & 3 \\ 3 & 1 & 2\end{array}\right) I,\left(\begin{array}{lll}1 & 2 & 3 \\ 1 & 2 & 3\end{array}\right) I\right\}$ is a subgroup of GI.

(b) $N(H)=T \cup S=\left\{\left(\begin{array}{lll}1 & 2 & 3 \\ 2 & 1 & 3\end{array}\right),\left(\begin{array}{lll}1 & 2 & 3 \\ 1 & 2 & 3\end{array}\right),\left(\begin{array}{lll}1 & 2 & 3 \\ 2 & 3 & 1\end{array}\right) I,\left(\begin{array}{lll}1 & 2 & 3 \\ 3 & 1 & 2\end{array}\right) I,\left(\begin{array}{lll}1 & 2 & 3 \\ 1 & 2 & 3\end{array}\right) I\right\}$ is an AH-subgroup of $\mathrm{N}(\mathrm{G})$.

(c) $\mathrm{N}(\mathrm{H})$ is AH-cyclic and AH-abelian subgroup, since T,S are cyclic and abelian.

(d) $\mathrm{N}(\mathrm{H})$ is AH-Nilpotent and AH-solvable, since T,S are nilpotent and solvable.

\section{Conclusion}

In this article, we have defined the concept of AH-homomorphism in a neutrosophic group for the first time. Also, we have introduced some corresponding notions such as AH-subgroup, AH-normailty, and AH-factors. Many examples and theorems were constructed to clarify the validity of these concepts.

Funding: "This research received no external funding"

Conflicts of Interest: "The authors declare no conflict of interest."

\section{References}

[1] Abobala, M., " n-Refined Neutrosophic Groups I", International Journal of Neutrosophic Science, Vol. 0, No. 1, pp.. 27-34, 2019

[2] Abobala, M., "n-Refined Neutrosophic Groups II", International Journal of Neutrosophic Science, Vol. 0, No. 1, pp. 47-56, 2019

[3] Agboola, A.A.A,. Akwu, A.D,. and Oyebo, Y.T,. "Neutrosophic Groups and Subgroups", International .J .Math.Combin, Vol. 3, pp. 1-9, 2012.

[4] Agboola, A.A.A., Akinola, A.D., and Oyebola, O.Y.," Neutrosophic Rings I ", International J.Math combin, Vol. 4, pp. 1-14, 2011. 
[5] Atanassov, K., "Intuitionistic Fuzzy Sets", Fuzzy Sets and Systems, 20(1), pp.87-96, 1971.

[6] Haushi, M., " Algebraic Structures 1", Tishreen University Press, pp. 102-209, 2005.

[7] Kandasamy, V.W.B., and Smarandache, F., "Some Neutrosophic Algebraic Structures and Neutrosophic NAlgebraic Structures", Hexis, Phonex, Arizona 2006.

[8] Smarandache, F., "Neutrosophy, Neutrosophic Probability, Set and Logic", Amer. Res Press, Rehoboth, USA, http://fs.gallup.unm.edu/eBook-neutrosophics6. pdf (sixth version). 1998.

[9] Smarandache, F., " Neutrosophic Set, A Generalisation of the Intuitionistic Fuzzy Sets", Inter. J. Pure Appl. Math., 24, pp. 287-297, 2005.

[10] Wang, H., Zhang, Y., Sunderraman, R., and Smarandache, F., "Single Valued Neutrosophic Sets, Fuzzy Sets, Rough Sets and Multivalued Operations and Applications", 3(1), pp. 33-39, 2011.

[11] Rotman, J., "The Theory of Groups", University of Illinois, Urbana, pp. 120-180, 1971. 